\title{
RESEÑA BIOGRÁFICA DEL DR. EDUARDO GASTRO MURILLO
}
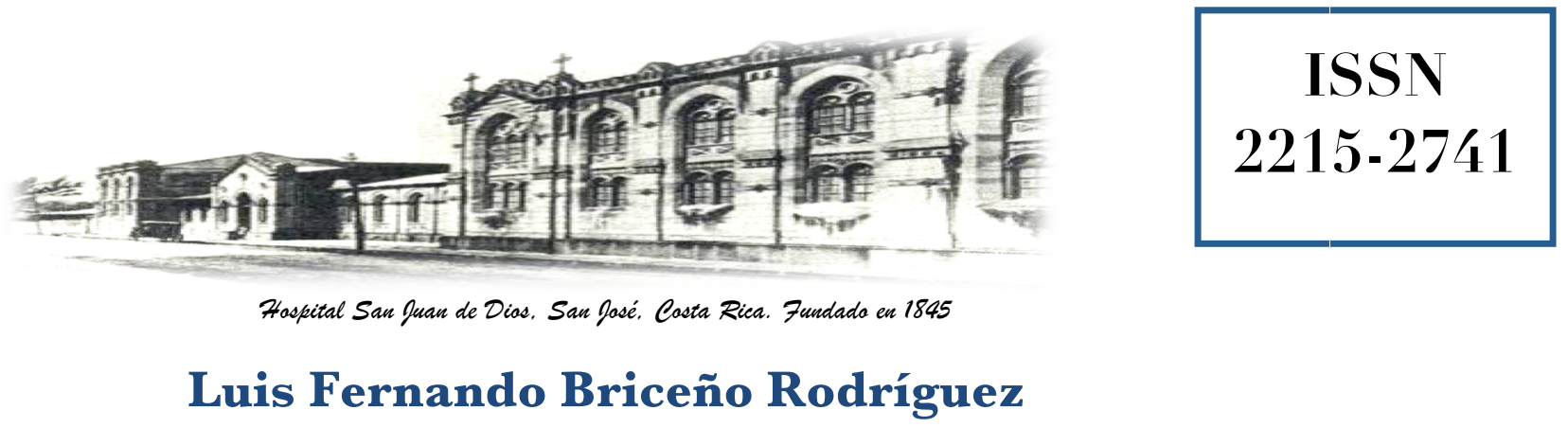

Recibido: $\quad 22 / 07 / 2013$

Aceptado: $\quad 14 / 08 / 2013$

HSJD

Luis Fernando Briceño Rodríguez ${ }^{1}$

\begin{abstract}
${ }^{1}$ Ex-Miembro Sección de Medicina - Hospital San Juan de Dios. Profesor Asociado Universidad de Costa Rica (pensionado). Correo electrónico: Ibriceno@racsa.co.cr
\end{abstract}

Como Profesor Universitario, el doctor Eduardo Castro Murillo fue declarado Profesor Emérito de la Escuela de Medicina de la Universidad de Costa Rica en el Hospital San Juan de Dios por su dedicación a la Docencia.

Nació en San José el 17 de noviembre de 1928 y falleció el 18 de enero del 2010, a los ochenta y un años de edad.

El doctor Castro M. fue un apasionado de la enseñanza de la Medicina en nuestro querido Hospital San Juan de Dios (HSJD), en la Universidad de Costa Rica (UCR), en la Sección de Medicina del HSJD y en el Posgrado de Medicina Interna del CENDEISSS.

Obtuvo su bachillerato de secundaria en el Liceo de Costa Rica. Doctor en Medicina en la Escuela de Medicina de la Universidad de El Salvador en 1958. Realizó su Internado Universitario y su Residencia en Medicina Interna en el HSJD.
Llevó a cabo el Internado en Medicina Interna en Temple University Hospital, Pensilvania - EUA.

Obtuvo el diploma del National Board of Medical Examiners de los EUA.

Posteriormente llevó a cabo estudios de Posgrado en Reumatología en el Hospital of the University of Pennsylvania, Philadelphia - USA y en Cornell Medical Center, New York - USA; y estudios de Posgrado en Inmunología en Louisiana State University, Louisiana - USA.

Recibió el premio The Educational Council Foreing Medical Graduates en Evanston Illinois - USA en 1963.

Fue Asistente Especialista en Medicina Interna y en Reumatología en la Sección de Medicina del HSJD durante 32 años, de 1968 a 2000. 
Realizó varias publicaciones científicas en Reumatología y en Inmunología.

El doctor Castro M. ocupó los cargos de Coordinador de Cátedra de Medicina y de Director del Departamento Clínico de la UCR en el HSJD.

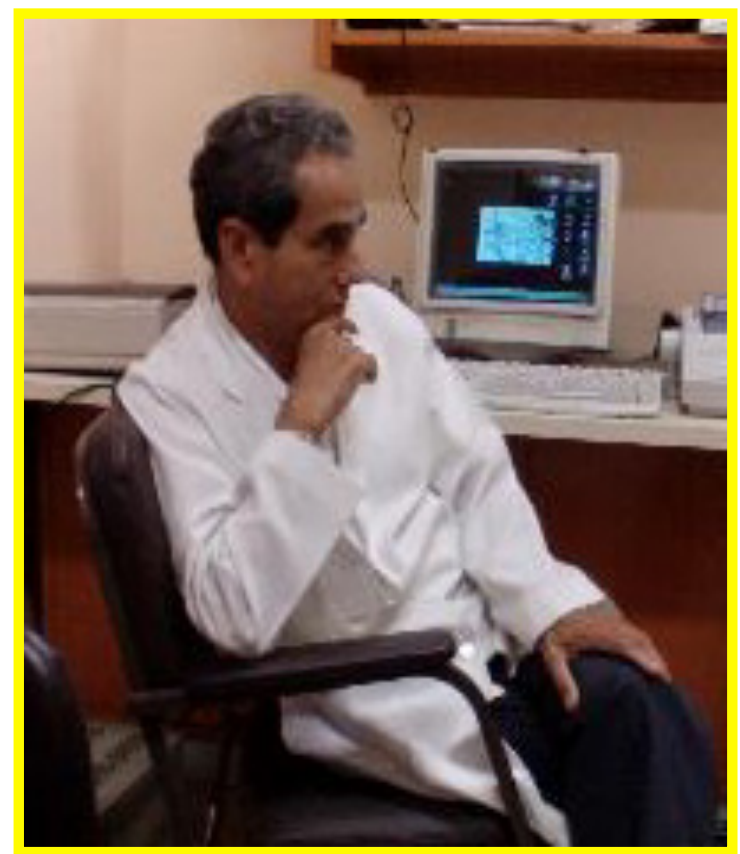

Imagen 1. Fotografía del doctor Eduardo Castro Murillo, en el Departamento de Patología del Hospital San Juan de Dios, durante la preparación de una de la Sesiones Anátomo-Clínicas de la Sección de Medicina.

Las graduaciones de estudiantes de la Escuela de Medicina de la UCR de 1980 y 1982 llevan su nombre.

Fue Coordinador de Docencia de la Sección de Medicina de 1982 a 2000, y Coordinador del Posgrado de Medicina Interna del CENDEISSS en el HSJD.

Desde 1985, el Aula de la Sección de Medicina del HSJD lleva su nombre, por su dedicación a la Docencia en la Sección.

El doctor Eduardo Castro Murillo fue el "Maestro" por excelencia, dedicando gran parte de su vida profesional a la enseñanza de la Medicina.
Inculcó en múltiples generaciones de estudiantes de pregrado, grado y posgrado, de médicos generales y de especialistas en Medicina Interna y de otras Especialidades del Área Médica, actitud, motivación y sobre todo, pensamiento crítico y analítico.

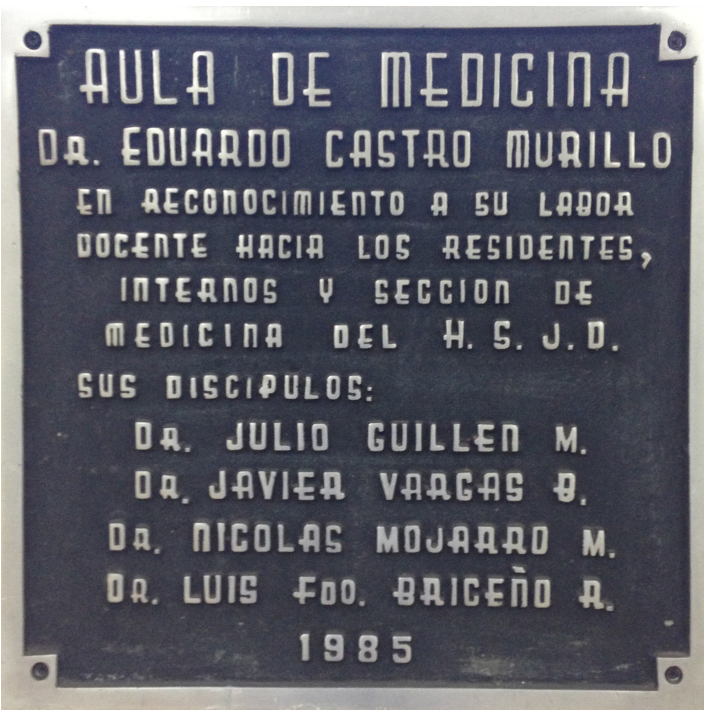

Imagen 2. Fotografía: Placa en homenaje al doctor Castro Murillo ubicada en el Aula de Medicina de la Sección de Medicina del Hospital San Juan de Dios, develada en 1985.

Aquellos que tuvimos la oportunidad de estar en sus clases, en sus discusiones de casos y en reuniones formales o informales, recordamos siempre su forma de transmitir conceptualmente sus conocimientos y sus puntos de vista.

Con su trabajo, inspiró durante muchos años a todos quienes le acompañamos en el camino del conocimiento.

Su visión y opinión sobre la docencia y la enseñanza de la Medicina siempre fueron críticas, respetuosas y acertadas.

Para el doctor Castro Murillo: "La docencia no consiste únicamente en la transmisión del conocimiento. La adquisición del conocimiento (decía) se puede lograr a través de los libros, de revistas médicas e Internet.

La docencia es lograr la motivación para descubrir la belleza que hay en la ciencia, en el conocimiento de la Medicina; porque realmente ese conocimiento encierra belleza”. 
La vida nos daría el privilegio de trabajar a su lado en la docencia médica durante más de quince años, habiendo concluido ambos, después de su retiro laboral, el libro "METODOLOGÍA DE ANÁLISIS CLÍNICO - Un enfoque didáctico en la enseñanza y práctica de la Medicina" en 2007.

Desde muy temprano en nuestra formación, el doctor Castro M. empezó a inculcarnos Análisis Clínico, como el análisis exhaustivo y cuidadoso de toda la información que se obtiene del paciente, y que nos permite entender apropiada e integralmente al enfermo y no sólo la enfermedad. Su práctica constante nos llevó a muchos a adquirir una destreza intelectual de gran valor.

Todos aquellos quienes le conocimos estamos muy agradecidos con él, a todos nos dejó un gran entusiasmo por nuestra profesión. A todos nos dejó grandes enseñanzas. 